\title{
A TIME PURIFIED/SEPARATED ANTIPROTON BEAM AT THE AGS
}

M. Bachman, a M. L. Barlett, a B. Bonner, $h$ S. Borenstein, $c$ D. Bridges, 9 H. Brown, $\mathrm{C}$ J. Buchanan, 1 J. C. Clement ${ }^{\prime}$ I. Daftari, $j$ R. Debbe, $A_{b}$ Deguzman,j R. W. Fergerson, $H$. Flick, W. Fickinger, $d$ L. Gray, G. W. Hoffinạn, a C. L. Hgllas, a T. Kalogeropoulos, J. Kruk, A. Macasero,j R. Marino, B. Mayęs, E. C. Milner, R. Moss, G. S. Mutchler, 1 L. Pinsky, P. J. Rjley, D. K. Robinson, d M. Sakitt, ${ }^{C}$ J. Skellly, ${ }^{C}$ L. G. Tang, ${ }^{\text {S. }}$. W. Xu, ${ }^{a}$ Y. Xue

auniversity of Texas at Austin; DPennsylvania State University at Bloomsburg; CBrookhaven National Laboratory; dCase Western Reserve

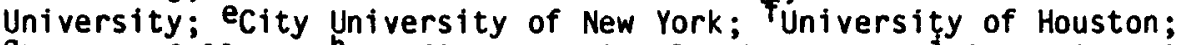

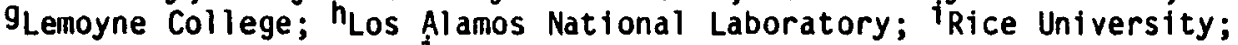
jSyracuse University.

\section{ABSTRACT}

A $1 \mathrm{~km}$ antiproton beam has been designed for construction at the AGS. The momentum band can be varied between $\pm 0.3 \%$ to $\pm 1.0 \%$, and the resolution for tagged particles will be $\delta p / p \sim 10^{-4}$ at beam rates as high as $10^{6} \overline{\mathrm{p}} / \mathrm{s}$. Separation by decay purification will be on the order of $1 \bar{p} / 10\left(\pi^{-}+\mu^{-}\right)$. This beam will be used in a detailed investigation of Charmonium including a measurement of the $x$ widths. We will also search for expected but as yet unseen states, and search for possible $I=1$ events which would imply the existence of four quark states. This facility will also lend itself to a wide variety of exciting physics such as the proton form factor including both $e^{+} e^{-}$and $r y$ final states, two-body hadron final states, antinucleus yields, and possibly tagged hadron beams (i.e., $\Lambda$, $\Xi$, etc.). When heavy ions become available at the AGS, one can measure various long lived particle yields. Finally, with as many as $10^{7}$ polarized muons in the beam, one has the possibility to use them for nuclear structure studies.

\section{INTRODUCTION}

We have recently submitted a proposal to BNL to construct a $1 \mathrm{~km}$ long secondary beamline for use primarlly as a $\bar{p}$ facility in the momentum range from $1-10 \mathrm{GeV} / \mathrm{c}$. The principle feature of this beamline is its length which will permit purification of the $\bar{p}$ beam by allowing time for $\pi$ decay. We refer to this as a Time Purified Bean (TPB). Complete time separation can also be achleved by leaving the AGS rf on during extraction which will bunch the incident primary beam on the production target in $<1$ ns pulses separated by a minimum of $220 \mathrm{~ns}$. The TOF differences as a function of particle mass will allow clear temporal separation at the downstream end of the beamline. We refer to this mode as a Time Separated Beam (TSB). The title Time Purified/Separated Beam (TP/SB) is used as a generic reference to the entire facility.

Also included in the design is a High Resolution Beamline Spectrometer (HRBS), which will be situated on the downstream por- 
tion of the beamline. This magnetic spectrometer will be capable of measuring $\delta p / p \sim 10^{-4}$. Further, since the total flux in the beam will be on the order of $10^{7} / \mathrm{sec}$ with as many as $10^{6} \frac{\bar{p}}{\mathrm{~s} e c}$, the HRBS will be equipped with real-time, high speed beam measurement and tagging instrumentation that will produce not only a particle identification "tag" for use in any downstream experiment, but also a momentum "tag" at the level of a $\delta p / p$ of $10^{-4}$. These tags can be provided on a particle by particle basis at rates exceeding $10^{6} / \mathrm{sec}$. The concept of TSBs and TPBS has been explored extensively over the last decade. Preliminary experiments in 1974 at the AGS 1 and subsequent studies 2,3 have demonstrated the fegsibility of operating in the TSB mode. In 1980 one of us (H. Brown) 4 completed a preliminary design for such a beam line at the AGS. This was followed in 1981 by an SPS proposal at CERN (Ferro-Luzzi et al.) ${ }^{5}$ for a similar $1 \mathrm{~km}$ antiproton beam specifically for use in studying Charmonium. This CERN proposal was strongly recommended, but the CERN management decided against its construction due to financial commitments to other projects such as LEAR and LEP, and the competing ISR experiment on Charmonium, R704.6 In consideration of these developments and studies, we now propose to bufld a beam suitable for both the study of very narrow states (e.g. Charmonium), and the systematic measurement of the $\overline{p p}$ interaction cross section.

\section{BEAMLINE DESIGN}

The basic idea of the TP/SB optics is to construct a quadrupole "objective lens" system, with short focal lengths, to gather antiprotons from the production target over a large solid angle and project (match) them into a long transport line composed of regularly spaced focusing and defocusing quads (a FODO line) which will then carry the beam, without loss, except by decay, to the experiment. A bending section must be embedded, efther in the matching section or in the FODO line, which will define a desirable momentum band for the beam.

The beamline is $1 \mathrm{~km}$ long and will collect and transport ps within the momentum range from $1-8 \mathrm{GeV} / \mathrm{C}$ wth the initial choice of magnets currently available at the AGS. The upper limit is determined by the fields in the front quads and the first bending magnet. Acquisition of higher field magnets in these front elements would allow the use of momenta to beyond $10 \mathrm{GeV} / \mathrm{c}$ where the production cross section is dwindling. The momentum band transported can be varied from $\pm 0.3 \%$ to about $\pm 1 \%$. Figure 1 shows the calculated $\bar{p}$ flux for the proposed beam as well as the total flux of $\pi^{-}$and $\mu^{-}$ remaining at the end of the beamline. Figure 2 shows the beam momentum resolution by (a) TOF down the beamline, (b) TSB rf structure, and (c) HRBS. These include the effects of beamline instrumentation.

In summary, this beam will provide $\bar{p}$ fluxes as high as $3 \times 10^{6} \bar{p} / s$ per $10^{12}$ protons on the production target, with a momentum resolution of $\delta p / p \sim 10^{-4}$. The $\pi^{-+\mu^{-}}$contamination will be reduced, achieving a total $\mathrm{p} /\left(\pi^{-}+\mu^{-}\right)$ratio $\sim 0.1$. Finally, in the TSB mode, one can achieve an absolute particle separation by TOF difference. 




p $|\mathrm{GN} / \mathrm{c}|$

Figure 1(a). Proton yields as a function momentio bite.

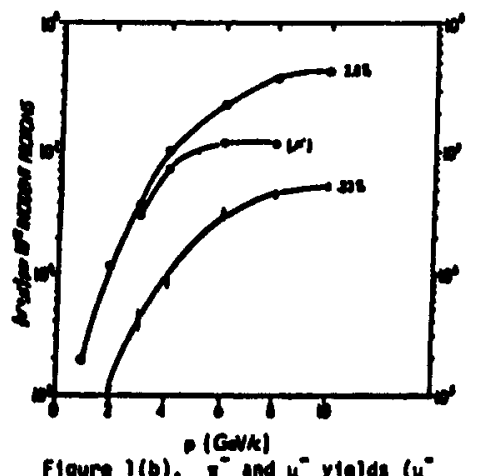

Figure $1(b) .-^{-}$and $\mu^{-}$ylelds $\left(\mu^{-}\right.$ is Por 2.85 incentin bitel.

\section{ANTIPROTON PHYSICS AT THE AGS}

Antiproton beans offer a wide range of experimental opportunitfes in many areas of particle physics: exotic atoms, nuclear potentials, meson spectroscopy, nucleon form factors, and others. In addition, they may provide unique opportunitles for tagged particle experiments. The physics potential in these flelds remains largely untapped.

The proposed facility would be unique in the region from 2 to 10 GeV/C. Below 2 GeV/c the LEAR factlity can produce very pure well-collimated $\bar{p}$ beans of high intensity $\left(10^{6} \overline{\mathrm{p}} / \mathrm{sec}\right)$. However, at present LEAR is heavily subscribed, and for the foreseeable future the beam time avallable per experiment w1ll be severely limited. Further, given the extremely narrow momentum bite of the LEAR beam $\left(8 P / p=10^{-3}\right)$, for experiments requiting broad ranges of



Figure 2. Momentum resolution including effects of beamline instrumentation. 
of incident momenta such as cross section searches, the TP/SB would be substantially superior to LEAR in the $1-2 \mathrm{GeV} / \mathrm{c}$ range.

The following sections discuss some of the interesting physics it would be possible to explore with a TP/SB at the AGS. Finally, it should be mentioned that most of the physics discussed can be accomplished in the TPB mode with the slow extracted beam.

1. Charmonium Physics

There are many fundamental problems regarding Charmonium, such as the existence of unseen states and measurement of widths. An intense antiproton beam at the AGS will be a unique opportunity to pursue this physics. Currently the concluding ISR experiment (R704) using a jet target and a cooled antiproton beam is looking for the $x_{2}$ and $n_{c}$ states via

$$
\begin{aligned}
\bar{p}+p & +x_{2}+\gamma+\psi\left(e^{+} e^{-}\right) \\
& +n_{c}+\gamma+\gamma
\end{aligned}
$$

In a test run 70 events background free including four $x_{2}$ events have been seen via $x_{2} \rightarrow \gamma+\psi+e^{+} e^{-}$. The principal objective is to measure the $x_{2}$ widths that provide a test of the two-gluon decay mechanism and QCD predictions as well as to measure $n_{c} s$. The limited time avallable and a resolution of $2 \mathrm{MeV}$ will not allow final conclusions for these very important measurements. The TP/SB would have a luminosity 100 times the maximum instantaneous luminosity attainable in that experiment. Furthermore, a mass resolution of $300 \mathrm{KeV}$ could be achieved in the TP/SB with a high-resolution spectrometer. The complete mapping and measurement of the Charmonium system is of crucial importance to QCD. It holds the prospect of being to this theory what the hydrogen atom was to Quantum Mechanics.

2. Spectroscopy

With the fluxes available at the proposed facility one should ultimately be able to conduct a high precision total cross section measurement and achieve a statistical accuracy of 15-25 $\mu \mathrm{b}$ in mass bins of $300 \mathrm{KeV} / \mathrm{c}^{2}$ from $1-10 \mathrm{GeV} / \mathrm{c}$ in 1000 hours of beam time. Here one also has the possibility to measure simultaneously with parallel hardware the large angle elastic, charge-exchange, and two-body anninilation cross sections. Phase II of our proposal includes a 500 hour inttial look at the total cross section from $1-8 \mathrm{GeV} / \mathrm{C}$. The other cross section measurements will be included in later proposals along with the investigation of any interesting structures seen during Phase II.

3. Electromagnetic Interactions

a. Proton Form Factor in the Time-Like Region

The proton form factor in the time-like region is poorly known; it can be measured through the reaction

$$
\bar{p}+p+e^{+}+e^{-}
$$

While there is an experiment at LEAR (PS170) to measure this reaction up to $2 \mathrm{GeV} / \mathrm{C}$ incident momentum, a TP/SB at the AGS would offer the only hope of extending this important measurement to higher 
(above $2 \mathrm{GeV} / \mathrm{c}$ ) energy. Because of the small cross section, the maximum possible $\bar{p}$ fluxes are required (at least $10^{6} \frac{\bar{p}}{\mathrm{p}} / \mathrm{pulse}$ ). A good map of the proton form factor at all energies will be an important test of strong interaction theories. Furthermore, if there are vector mesons coupled strongly to $\overline{\mathrm{pp}}$, they will show up in this form factor and thus supplement the $\mathrm{e}^{+} \mathrm{e}^{-}+$hadron physics.

b. Two Photon Physics

The intriguing Compton hard scattering in the time-like region has not been studied, although a few events have been seen at low $\bar{p} p$ mass in the two-photon studies at PETRA. A good measurement of the cross section for

$$
\bar{p}+p+\gamma+\gamma
$$

from the lowest to the highest possible energies is of significant interest. Event rates are expected to be similar to $\bar{p} p+e^{+} e^{-}$; therefore, the reaction can be studied up to $4 \mathrm{GeV} / \mathrm{c}^{2} \mathrm{c} . \mathrm{m}$. energy at the TP/SB. Besides revealing proton form factors, this reaction provides information about $\mathrm{C}=+1$ states.

$$
\text { c. 1-2 GeV Spectroscopy }
$$

As an example of what antiprotons can do to further this field, we suggest exploration of vector and scalar mesons via the reactions

$$
\begin{aligned}
& p+\bar{p}+\pi^{0}+V\left(+e^{+} e^{-}\right) \\
& p+p+\pi^{0}+S(+\gamma \gamma)
\end{aligned}
$$

An old experiment at CERN 8 with $10^{3} \overline{\mathrm{p}}$ annihilations/sec at rest had revealed structure at $1.5 \mathrm{GeV} / \mathrm{c}$ via $\mathrm{pp}+\pi^{0}+\mathrm{V}\left(\rightarrow \mathrm{e}^{+} \mathrm{e}^{-}\right)$. Clearly, an increase of $10^{2}-10^{3}$ in $\bar{p}$ flux and present detector technology would allow an improved investigation of this phenomenon.

4. Tagged Beams

Tagged beams of $\pi^{0}, K^{+-}, K_{\ell}, \bar{n}^{9}, \Lambda, \bar{\Lambda}, Y, \bar{Y}, \Xi, \bar{\Xi}$, etc. can be made with an intense antiproton beam. Rates and experiments have been discussed elsewhere. 10 The physics possibilities range from a search for neutrino masses $\left(\pi^{0}+2\right.$ neutrinos) to searches for rare or forbidden decays, measurements of absolute decay rates, improved measurements of $C P$ parameters, searches for $\pi^{0}+3 \gamma$, extended limits on CPT, and a search for CP violation in $(\Lambda, \bar{\Lambda})+\left(p \pi^{-}, p \pi^{+}\right)$decays. However, all these possibilities become most attractive as the AGS proton intensity avallable for this beam reaches more than $10^{13} \mathrm{p} / \mathrm{s}$. At these fluxes the TSB mode of operation becomes more attractive.

With current AGS flux of approximately $10^{12} \mathrm{p} / \mathrm{sec}$, tagged ant 1 neutrons will be quite feasible. Fluxes of approximately $10^{4}-10^{5}$ $\bar{n} / \mathrm{sec}$ will be available via the $\bar{p} p \rightarrow \bar{n} n$ reaction. A complete physics program with antineutrons can thus be implemented without the complexity introduced by deuterium targets.

5. Antideuterons

The measurements at the AGS by Dorfan et al.11 of antideuteron production between 4.5 and $6 \mathrm{GeV} / \mathrm{c}$ indicate that the proposed beam will include approximately 100 antideuterons/pulse. These antideuterons can be used in interactions with nuclei to simulate heavy ion collisions by depositing $4 \mathrm{GeV}$ in the form of low energy pions 
in the localized region of the nucleus. In fact, if there are measurable fluxes of $3 \overline{H e}$ and $3 \bar{H}$, one may be able to use those as well.

In conclusion, we believe there is a rich and unique program of physics to be explored with a $\bar{p}$ beam at the AGS in the region between $1-10 \mathrm{GeV} / \mathrm{C}$.

\section{OTHER AREAS}

1. Heavy Ion Physics

The TP/SB which is ideal for studying particle yields, can be used to measure $p, d, t$ yields, or other long-lived isotopes, in heavy ion collisions. When heavy ions become available at the TP/SB production target, we plan to submit a proposal to study the resulting yields.

As can be seen in Fig. 1 as many as $10^{7}$ muons/1012 protons will be present at the end of the TP/SB. These muons are polarized and more desirable to work with than electrons whtch produce large bremsstrahlung background. As such, they may provide an attractive beam for nuclear structure studies.

\section{SUMMARY}

We have proposed the construction of new beamline at the AGS which we feel will become an essential facility over at least the next decade. The physics prospects are so exciting that beyond the Charmonium experiments, we anticipate severe competition for its use.

tWork supported in part by the U.S. Department of Energy. *Work supported in part by the National Science Foundation.

\section{REFERENCES}

1. A. Fainberg and T. E. Kalageropoulos, BNL-18938 (1974) (unpublished).

2. M. Q. Barton, BNL-19076 (1974) (unpublished).

3. T. Brando et al., Nucl. Inst. Meth. 180, 461 (1981).

4. H. Brown, BNL, EP\&S Division Technical Note No. 90 (1980).

5. CERN/SPSC 81-11; SPSC/P 157 (1981).

6. P. Litchfield. Proc. of the Third LAMPF II Workshop, LA-9933-C, Vol. 1, p. 344 (1984).

7. R. Barbiert et al., Phys. Lett. 61B, 464 (1976), and Phys. Lett. 95B, 93 (1980), as well as Ref. 6.

8. G. Bassompierre et al., Phys. Lett. 68Bk, 477 (1977).

9. B. W. Mayes, L. S. Pinsky and G. S. Wuitchler, Proc. of the III LAMPF-II Workshop, LA-9933-C, Vol. II, p. 738 (1983).

10. T. Kalogeropoulos, Proc. of the 2nd LAMPF II Workshop, LA-9572-C, Vol. II, p.619.

11. D. E. Dorfan, J. Eades, L. M. Lederman, W. Lee and C. C. Ting; Phys. Rev. Lett. 14, 1003 (1965). 